\title{
The Giant Star Ca II Ionization Problem: Mass Loss Revisited
}

\author{
Graham M. Harper, Alexander Brown, Thomas Ayres, and \\ Center for Astrophysics and Space Astronomy, University of Colorado, \\ Boulder, CO 80309-0389, USA
}

\author{
Stuart A. Sim
}

Imperial College London, Blackett Laboratory, UK

\begin{abstract}
Pioneering studies of winds from non-coronal evolved latetype stars were plagued by uncertainties in the $\mathrm{Ca}$ ionization balance which severely limited the accuracy of derived mass-loss rates. Here we re-examine the Ca II ionization balance in these stellar winds using FUSE spectra which reveal, for the first time, the flux from the photoionizing radiation field shortward of $1045 \AA$. We present a FUSE $912-1185 \AA$ spectroscopic survey of evolved late- $K$ and $M$ stars; including the $M$ giants $\alpha$ Cet (M1.5 III), $\gamma$ Cru (M3.5 III), $\beta$ Gru (M4.5 III), and R Dor (M8e III). Using FUSE spectra of $\alpha$ Tau (K5 III), supplemented with partial redistribution calculations of $\mathrm{H} \mathrm{Ly}-\alpha$ and $\mathrm{Ly}-\beta$, together with $\mathrm{UV}$ and radio data, we present a study of $\alpha$ Tau's wind ionization balance and derive new constraints which place the mass-loss rate significantly below that suggested by the Reimers formula.
\end{abstract}

\section{Introduction}

Mass loss from cool giants and supergiants is important for understanding the chemical evolution of the Galaxy. Stellar winds return nuclear-processed material from the dredge-up phases back into the ISM where it becomes available for the next generation of stars. Mass loss is also important for the stellar evolution of intermediate and massive stars; in particular, it controls the frequency of supernovae, and the amount of iron-peak and r-processed elements returned to the ISM. Mass-loss rates for late-type stars are, however, very difficult to determine, and the mechanisms that drive mass loss are poorly understood.

Early optical studies of mass loss from cool stars relied heavily on the Ca II $\mathrm{H} \& \mathrm{~K}$ wind absorption features as diagnostics. It was soon realized, however, that calcium exists as Ca III in the inner wind. Also at that time the UV $(1200<$ $\lambda(\AA)<3200)$ and FUV $(912<\lambda(\AA)<1200)$ photoionizing radiation fields were essentially unknown, and this led to an inherent uncertainty in derived mass-loss rates. Since 1978, IUE and HST have provided UV spectra for cool stars, but the photoionizing flux for the Ca II $4 s^{2} S$ ground state $\lambda<1044 \AA$ remained unknown until the Far Ultraviolet Spectroscopic Explorer (FUSE) provided a window into the FUV. 


\section{FUSE Observations}

Surprisingly few FUSE observations of non-coronal evolved late-type stars have been made, and most of these are from FUSE programs by the authors. The spectra presented here (Table 1) were retrieved from the Multimission Archive at Space Telescope, re-calibrated with CalFUSE v2.1.6, and custom extracted. Figure 1 shows the LiF $1000-1185 \AA$ spectra where the stellar signal and FUSE sensitivity are highest, for the sample of $\mathrm{K}$ and $\mathrm{M}$ giants - $\mathrm{R}$ Dor shows no significant signal. These spectra were obtained during orbital night when the airglow and geocoronal emission is weak. Surface fluxes are computed using the angular diameters adopted in Table 1. (Bedding, Robertson, \& Marson 1994; Bedding et al., 1997; Mozurkewich et al., 1991.)

Table 1. FUSE Sample of Non-Coronal Evolved Late-Type Stars. UD-uniform, LD-limb darkened disk. †More observations scheduled.

\begin{tabular}{lcccc}
\hline Star & Spec. Type & $\begin{array}{c}\text { Angular Diam. } \\
\text { (mas) }\end{array}$ & $\begin{array}{c}\text { Exposure } \\
(\mathrm{ksec})\end{array}$ & FUSE ID \\
\hline & & & & \\
$\alpha \mathrm{Tau}$ & K5 III & $21 \mathrm{LD}$ & 12 & $\mathrm{P} 1040901$ \\
$\alpha \mathrm{Cet}$ & M1 III & 13 LD & $10 \dagger$ & C0230303 \\
$\gamma$ Cru & M3 III & $23 \mathrm{UD}$ & 23 & $\mathrm{~B} 0680101$ \\
$\beta$ Gru & M5 III & 27 UD & 21 & $\mathrm{C} 0230101$ \\
R Dor & M8 III & 57 UD & $38 \dagger$ & C0230203
\end{tabular}

A prominent feature in the $\mathrm{SiC}$ channels is the C III $977 \AA$ resonance line, which is observed as late as M3 III ( $\gamma \mathrm{Cru})$. The most prominent emission features in the LiF channels are the forest of narrow lines between 1106-1150 first observed in ORFEUS SPAS-II spectra by Dupree \& Brickhouse (1998). Most lines were identified from high S/N and R 18,000 FUSE spectra by Harper et al. (2001) as fluorescent Fe II pumped by $\mathrm{H}$ Ly $\alpha$ from the excited $\left(a^{4} D\right)$ term. The rise in the chromospheric continuum shortward of $1100 \AA$ marks the $\mathrm{C} \mathrm{I}{ }^{3} P$ ground state edge. The photoionization of the Ca II ground state is controlled by the $\mathrm{C}$ I continuum and $\mathrm{H} \operatorname{Ly} \beta(\lambda 1026 \AA)$. An early discovery with FUSE was the detection of collisionally excited O VI $(\lambda 1032, \lambda 1038 \AA)$, a proxy for coronal emission, in $\alpha$ Tau (K5 III). There are features close to these wavelengths in $\gamma$ Cru (M3 III), however, there are other unidentified features in the structured continuum and identification is ambiguous.

\section{Ca II/Ca III Ionization Balance}

To re-evaluate the mass-loss rates derived from optical studies of $\mathrm{Ca}$ II wind absorption features, we first quantify the Ca II/Ca III ratio. Two routes for photoionization of Ca II are $\mathrm{H} \mathrm{Ly} \beta$ and the $\mathrm{C}$ I continuum from the ground state, 


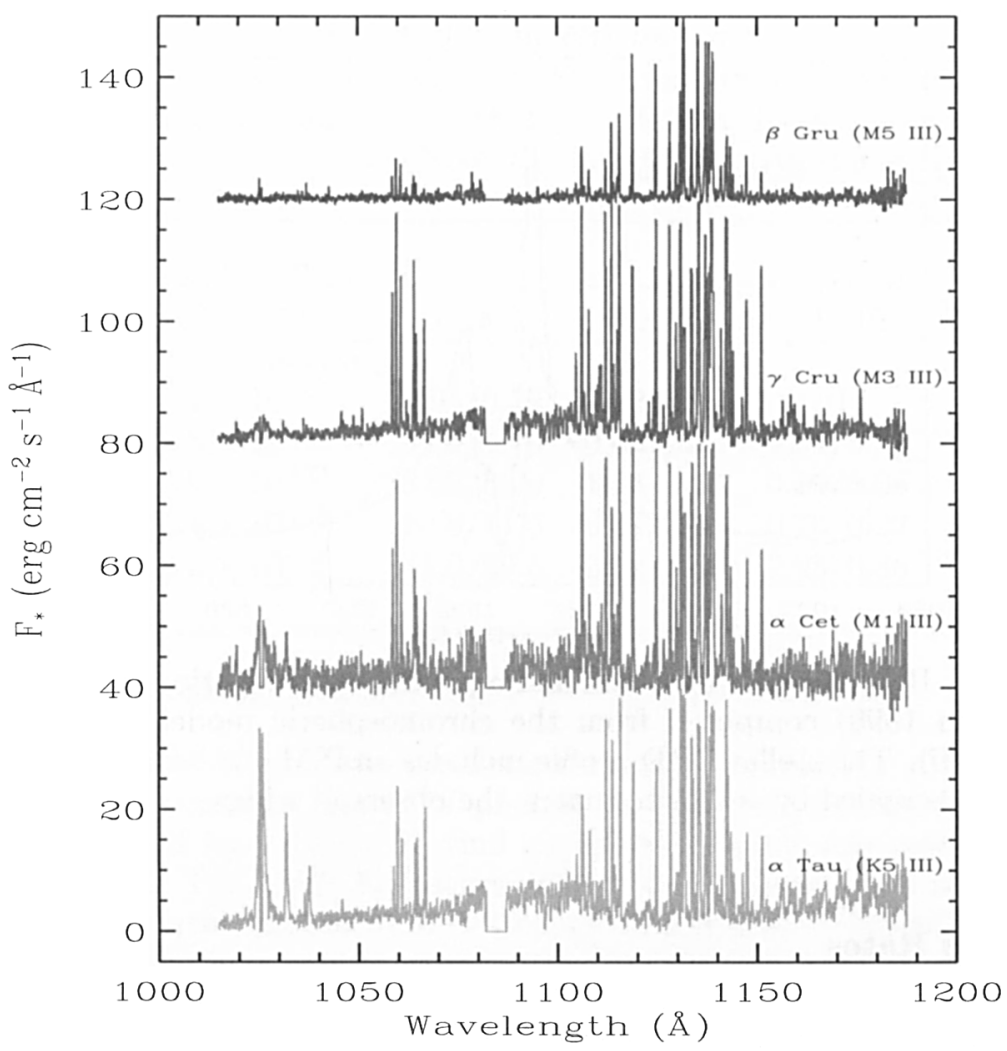

Figure 1. FUSE orbital night LiF spectra for the $\mathrm{K}$ and $\mathrm{M}$ giants, plotted as surface fluxes (each offset by 40). The break in data near $1080 \AA$ is from the space between the detector segments. The strongest fluorescent Fe II emission lines between 1106-1150 $\AA$ have been clipped for clarity. These lines and the Cr II lines near $1060 \AA$ are pumped by $\mathrm{H}$ Ly $\alpha$ from excited levels, and hence appear shortward of $1215 \AA$. The rise shortward of C I continuum edge at $1100 \AA$ is clearly seen.

and H Ly $\alpha$ from the metastable $3 d^{2} D$ term - both Lyman lines lie just shortward of the bound-free edges. To obtain photoionization rates the $\mathrm{H}$ Lyman profiles must be reconstructed to remove the effects of the ISM and geocoronal emission. Figure 2 shows $\alpha$ Tau's H Ly $\beta$ profile and a scaled PRD line profile computed (Sim 1999) from the detailed chromospheric model of McMurry (1999).

The photoionization cross-section edge for the metastable term is $6.1 \times$ $10^{-18} \mathrm{~cm}^{2}$ at $1218 \AA$ which is a factor of 30 greater than for the ground state at $1044 \AA$ (Norcross, in Shine \& Linsky, 1974). For $\alpha$ Tau the photoionization rate from the metastable level is $\sim 7000$ times that of the ground state. In the cool low density giant winds, the metastable level is populated by the scattering of chromospheric $\mathrm{Ca}$ II $\mathrm{H} \& \mathrm{~K}$ photons into the infrared triplet, and by direct photospheric pumping by the forbidden lines at $7291 \& 7324 \AA$. H Ly $\alpha$ is extremely effective in ionizing Ca II to Ca III even in the cool winds of M giants. 


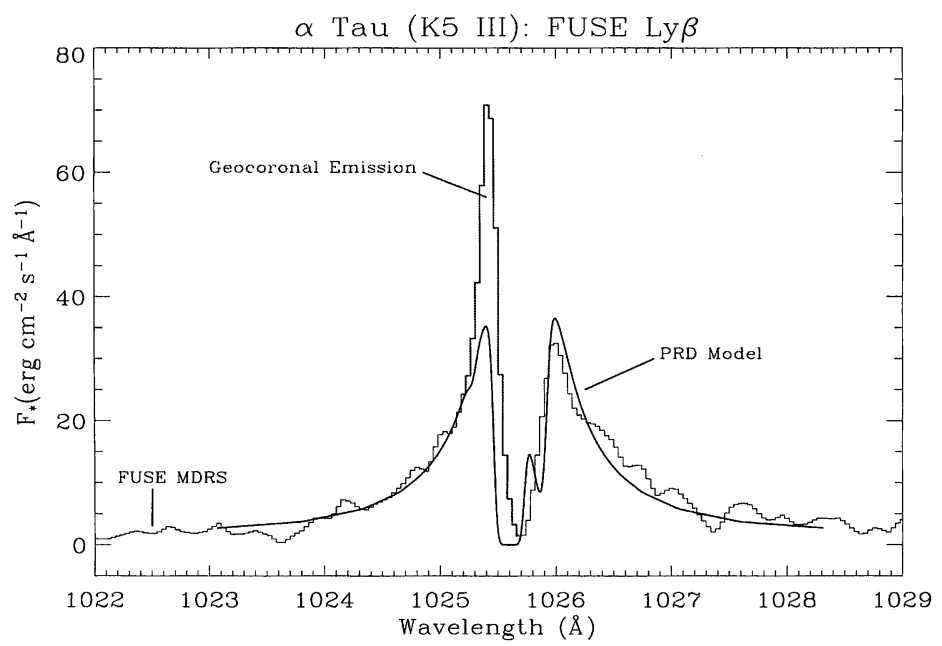

Figure 2. $\quad \mathrm{H} \mathrm{Ly} \beta$ profile of $\alpha$ Tau and a partial redistribution (PRD) profile (Sim 1999) computed from the chromospheric model of McMurry (1999). The stellar PRD profile includes an ISM and wind+ISM model and is scaled by $\times 1.15$ to match the observed wings.

\section{Mass-Loss Rates}

K5 III marks the onset of circumstellar (CS) Ca II H \& K absorption amongst giant stars, and $\alpha$ Tau provides a test-case to establish the mass-loss rates required for CS Ca II H \& K to be detectable. In $\alpha$ Tau the CS features are variable and not always present (Reimers 1977); when present they can have optical depths of $\tau \sim 0.5$. To the simplest approximation the following parameters are required to describe the formation of Ca II H \& K CS features:

(1) Ca II photoionization rates; $(2) V(R)$ wind velocity as a function of radius;

(3) $\dot{M}$ mass-loss rate; (4) $T_{e}=T_{e}\left(R_{0}\right)\left(R_{0} / R\right)^{p}$ parameterized wind temperature;

(5) $x=n_{H I I} / n_{H}$ hydrogen ionization at the base of the flow.

For $\alpha$ Tau, FUSE and HST spectra now tightly constrain (1); Robinson, Carpenter \& Brown (1998) have derived estimates for (2) and (3) (1.4 $\times$ $10^{-11} \mathrm{M}_{\odot} \mathrm{yr}^{-1}$ : P Cygni models), and (4) is weakly constrained by wind absorption features in Fe II line profiles and cm-radio observations. The Ca II/Ca III balance at the wind base is set by (1), (4), and (5).

Using these data, the optical depth for the Ca II K CS line is computed for different initial values of $x$ at $R=1.2 R_{*}$. For the low mass-loss rate indicated for $\alpha$ Tau the hydrogen ionization is frozen-in, so that the electron density is a function of $\dot{M}$ and $x$. Adopting an excitation temperature for the metastable term of $T_{e x c}=4000 \mathrm{~K}: y=n_{C I I} / n_{C} \ll 0.01$ and $n_{C I I} / n_{C I I I} \ll 1$. Using analytical solutions for steady advection for the $\mathrm{H}$ and $\mathrm{Ca}$ ionization balance we compute the maximum optical depth $\left(\tau_{\lambda}\right)$ for the Ca II K CS line, shown in Table 2, for two different values of the wind cooling parameter $p$. The solutions show that $y$ slowly increases with radius showing no sharp change reminiscent of a distinct shell. 
Table 2. Maximum $\tau_{\lambda}$ Ca II K CS feature for different initial hydrogen ionization fractions with $T_{e x c}=4000 \mathrm{~K}$. Data pairs for different $T_{e}$ models: $\mathrm{p}=1.33 / 0.50$. Note $T_{\text {exc }}=3000 \mathrm{~K}$ gives similar results.

\begin{tabular}{ccccc}
\hline & \multicolumn{4}{c}{$x\left(1.2 R_{*}\right)=n_{H I I} / n_{H}$} \\
Mass-loss Rate \\
$\mathrm{M}_{\odot} \mathrm{yr}^{-1}$ & 0.1000 & 0.0100 & 0.0010 & 0.0001 \\
\hline & & & & \\
$1.4 \times 10^{-11}$ & $0.94 / 0.32$ & $0.12 / 0.03$ & $0.01 /-$ & $-/-$ \\
$2.8 \times 10^{-11}$ & $3.06 / 1.16$ & $0.46 / 0.14$ & $0.04 / 0.01$ & $-/-$ \\
$5.6 \times 10^{-11}$ & $8.62 / 3.90$ & $1.74 / 0.54$ & $0.19 / 0.06$ & $0.02 / 0.01$ \\
$1.1 \times 10^{-10}$ & $20.6 / 11.6$ & $6.32 / 2.08$ & $0.75 / 0.22$ & $0.08 / 0.01$ \\
$2.2 \times 10^{-10}$ & $44.0 / 29.5$ & $21.2 / 7.69$ & $2.95 / 0.88$ & $0.31 / 0.09$ \\
& & & & \\
\hline \hline
\end{tabular}

\section{Discussion}

FUSE observations allow us, along with a reconstruction of the $\mathrm{H} \operatorname{Ly} \beta$ profile, to tightly constrain the FUV photoionization radiation field. Along with UV spectra of $\mathrm{H} \mathrm{Ly} \alpha$ the Ca II wind ionization problem can now be quantitatively addressed. For a CS K feature with $\tau \sim 0.5$ acceptable solutions from our simplified treatment have $\dot{M} \sim(0.1 / x)^{1 / 3} \cdot 1.4 \times 10^{-11} \mathrm{M}_{\odot} \mathrm{yr}^{-1}$ (Table 2).

Our findings can be summarized as: (a) In $\alpha$ Tau's steady wind Ca II does not form in a well defined shell. (b) Formation of Ca II CS features with discrete absorption near the terminal velocity requires an outward decrease in temperature. (c) The mass-loss rates required for Ca II CS features may be larger than derived from P-Cygni profiles. However, these values are an order of magnitude lower than those extrapolated from Reimers mass loss formula. New constraints on the hydrogen ionization at the base of the wind are required to further restrict the range of possible $\dot{M}$ solutions.

Acknowledgments. Research funded by grant NASA LTSA NAG5-4804 and FUSE grants NAG5-10339 \& NAG5-12438.

\section{References}

Bedding, T. R., Robertson, J. G., \& Marson, R. G. 1994, A\&A, 290, 340

Bedding, T. R., et al. 1997, MNRAS, 286, 957

Dupree, A. K. \& Brickhouse, N. S. 1998, ApJ, 500, L33

Harper. G. M., et al. 2001, ApJ, 551, 486

McMurry, A. D. 1999, MNRAS, 302, 37

Mozurkewich, D., et al. 1991, AJ, 101, 2207 VIII, 369

Reimers, D. 1977, A\&A, 57, 395

Robinson, R. D., Carpenter, K. G., \& Brown, A. 1998, ApJ, 503, 396

Shine, R. A., \& Linsky, J. L. 1974, Sol. Phys. 39, 49

Sim, S. A. 1999, MNRAS, 326, 821 